\title{
Capturing spacecraft attitude tracking control with angular velocity constraint
}

\section{Opinion}

On-Orbit Capture (OOC) technology is a key component of on-orbit servicing, which has garnered a great deal of researcher's attention in recent years, due to its potential application in future space missions, e.g., on-orbit assembly, on-orbit maintenance, and active debris removal. Various of space debris have been cast into Earth's orbit within the past sixty years, it increasing the collision risk posed to low-earth-orbit space missions; removing the debris and pushing it into Earth's atmosphere is an interesting potential solution to solve the problem. During the process of spacecraft on-orbit capture debris, the most important challenges are that the inertia moment of capturing spacecraft is changing and has a mutation, and the angular velocity is bounded due to the physical constraint. To address the issue of uncertainties with unknown parameters, adaptive technique and intelligent approach method are widely used to estimate the uncertainties. Previous researchers derived a class of adaptive attitude controllers, in which on-line parameter estimators are employed, for spacecraft, another researchers developed an adaptive output feedback attitude controller based on the on-line update law for spacecraft. To improve the convergence time, researchers proposed a finite-time adaptive attitude tracking controller for rigid spacecraft with inertia uncertainties. Yeh proposed a sliding-mode adaptive attitude controller for a spacecraft with a time-varying inertia matrix. In another paper, a low-frequency learning and fast adaptive controller was proposed for nonlinear, uncertain dynamic systems. Normalized input neural networks and Chebyshov neural networks have been applied to estimate the uncertainties, for example. The fuzzy engine has also been used to approximate the uncertainties of plants. Although these control schemes can be used to solve the attitude control problem with uncertain inertial matrix, they are very difficult to apply in practice due to the complexity of the controller and constraints. In practical situations, all the actuators and sensors have amplitude and rate limitations. The problem of spacecraft attitude control with bounded angular velocity and control saturation has attracted considerable
Volume 4 Issue 2 - 2018

\author{
Yin Chun Wu \\ School of Information and Control Engineering, Xi'an University \\ of Architecture and Technology, China
}

Correspondence: Yin Chun Wu, School of Information and Control Engineering, Xi'an University of Architecture and Technology, China, Email yincwxa2013@mail.nwpu.edu.cn

Received: February 10, 2018 | Published: April 04, 2018

interest to researchers. A saturated proportional-derivative control law was proposed for attitude control, it's only considered the control saturation, the parameter uncertainties and angular velocity saturation were not taken into account. Other researchers considered the attitude tracking problem with model uncertainties and control saturation, but did not account for angular velocity constrain. Considering the angular velocity constraints, a back stepping attitude controller was developed; an adaptive controller is designed, a barrier Lyapunov function was employed to prevent angular velocity from violating the angular velocity constraint. Similar methodology was applied in another study, but the control input saturation was not achieved due to the design of the barrier Lyapunov function. Considering the angular velocity and control input constraints, a novel robust nonlinear controller was presented by other researchers; however, the main contribution of their paper was the control allocation under redundant actuators (without considering the model uncertainties).

\section{Acknowledgements}

None.

\section{Conflict of interest}

The author declares there is no conflict of interest. 